\title{
The Quantum Measurement Problem and Cluster Separability
}

\section{P. Hájíček}

Received: 12 January 2010 / Accepted: 1 September 2010 / Published online: 15 September 2010

(C) Springer Science+Business Media, LLC 2010

\begin{abstract}
A modified Beltrametti-Cassinelli-Lahti model of the measurement apparatus that satisfies both the probability reproducibility condition and the objectification requirement is constructed. Only measurements on microsystems are considered. The cluster separability forms a basis for the first working hypothesis: the current version of quantum mechanics leaves open what happens to systems when they change their separation status. New rules that close this gap can therefore be added without disturbing the logic of quantum mechanics. The second working hypothesis is that registration apparatuses for microsystems must contain detectors and that their readings are signals from detectors. This implies that the separation status of a microsystem changes during both preparation and registration. A new rule that specifies what happens when these changes occur and that guarantees the objectification is formulated and discussed. A part of our result has certain similarities with 'collapse of the wave function'.
\end{abstract}

Keywords Quantum measurement problem · Registration apparatus · Detectors · Identical particles

\section{Introduction}

Discussions about the nature of quantum measurement were started already by founding fathers of the theory, persisted throughout and seem even to amplify at the present time.

An old approach to the problem of quantum measurement is Bohr's (its newer, rigorously reformulated version is Refs. [1, 2]). This approach denies that measuring apparatuses, and all classical systems in general, are quantum systems in the

P. Hájiček ( $₫)$

Institute for Theoretical Physics, University of Bern, Sidlerstrasse 5, 3012 Bern, Switzerland

e-mail: hajicek@itp.unibe.ch 
sense that all their properties can be derived from, or are compatible with, quantum mechanics. They must be described by other theories, called pretheories. Of course some classical properties of macroscopic systems can be obtained by quantum statistics. References [3, 4] show that such occasional applications of quantum mechanics to classical systems are compatible with the form of denying the universality of quantum mechanics specified there.

Modern approaches assume the universality of quantum mechanics together with various further ideas. An example is the quantum decoherence theory [5, 6], another the superselection sectors approach [7, 8], etc. However, the problem is far from being satisfactorily solved by any of the modern theories. Analysis of Refs. [9-11], as well as of our previous papers $[12,13]$, give an account of their shortcomings. In the present paper, we adopt the definition of the problem and the proof that it is far from being solved from Ref. [10].

Our starting point is the realist interpretation of quantum mechanics of Ref. [12] as well as the quantum theory of classical systems of Refs. [12, 13]. To solve the quantum measurement problem, additional ideas seem necessary and we propose some such ideas in the present paper. They might work in general, but we consider here only a special case. First, we assume the validity of non-relativistic quantum mechanics. Second, we restrict ourselves to measurements performed on microsystems such as elementary particles or systems composite of few particles. There are other systems on which recently a lot of interesting experiments have been done, such as Bose-Einstein condensates, strong laser beams or currents in superconductor rings. Such quantum states of 'large' systems, sometimes even macroscopic, will be ignored here. Third, we shall work within a simplified theoretical model of measurement due to Beltrametti, Cassinelli and Lahti [14]. Fourth, our theory will consider only those registrations in which the reading of registration apparatus is an electronic signal from a detector.

The main idea of the paper is a new assessment of the role that the existence of indistinguishable microsystems plays in general methods of quantum mechanics and in the special case of preparation and registration processes. Quantum systems can be divided into two classes according to the method of their description. First, there are particles and systems composite of particles of different type. Any of these and of their subsystems is a full-fledged quantum system $\mathcal{S}$ possessing a Hilbert space $\mathcal{H}_{\mathcal{S}}$. $\mathcal{H}_{\mathcal{S}}$ determines set of states $\mathcal{T}\left(\mathcal{H}_{\mathcal{S}}\right)_{1}^{+}$(positive operators with trace 1) and set of effects $\mathcal{L}\left(\mathcal{H}_{\mathcal{S}}\right)_{\leq 1}^{+}$(positive operators with norm bounded by 1 such as projections) from which its observables are constructed (for details, see $[1,2,10]$ ). The existence of this description and its physical meaning enabled us in Ref. [12] to view $\mathcal{S}$ as a physical object. Indeed, $\mathcal{S}$ has a sufficient number of objective properties because e.g. any element of $\mathcal{T}\left(\mathcal{H}_{\mathcal{S}}\right)_{1}^{+}$can serve as a prepared state of $\mathcal{S}$, and is then an objective property of $\mathcal{S}$ (for discussion, see Refs. [12, 13]). However, there are also systems composite of more than one particle of the same type. Then, there is only a common formal oneparticle Hilbert space from which a physical Hilbert space, states and observables of the whole system are constructed. Only the observables of the whole system are measurable. Thus, while the whole system is an object, none of the particles is. They are described in a different way, we call them subobjects and they form the second class of quantum systems. 
Only few textbooks (such as Ref. [15]) mention that these two modes of description contain a germ of contradiction (even without realist interpretations). Indeed, if we realise that the world is composite of many particles and that particles of each type occur in a huge number, then the justification of description of any such particle as an object, i.e., as if there were no other particles of the same type, seems to be strange. Nevertheless, such description can be justified and one justification is based on the idea of cluster separability of Ref. [15], p. 128. We reformulate this idea, introduce the notion of separation ${ }^{1}$ status, such as that of object or subobject, and find that there are consequences which can have some bearing on the quantum measurement problem.

If one applies the rules of ordinary quantum mechanics to microsystems that change their separation status, one can obtain wrong results. The theory cannot be expected to give reliable predictions in these cases. Our strategy in dealing with this problem will be first to calculate as if the ordinary quantum mechanics were applicable and then to see whether the observational evidence suggests any corrections. From the formally logical point of view, the current version of quantum mechanics ought to be understood as a theory of systems that have a fixed separation status and is thus incomplete. Hence, there is a possibility to add new rules to it without interference with its own notions and rules.

The plan of the paper is as follows. Section 2 summarises the BeltramettiCassinelli-Lahti model, defines the quantum-measurement problem and sketches a simple no-go theorem, using ideas of Ref. [10]. Section 3 analyses experiments with microsystems in order to motivate the assumption that reading of any real registration apparatus is a signal from a detector. This makes registration processes nearer to practice and, more importantly, it allows us to show that a microsystem changes its separation status during registration.

Section 4 reformulates the idea of cluster separability of Ref. [15] in more rigorous terms. This facilitates the introduction of the key notions of the paper: the separation status of a microsystem and its changes. In Sect. 5, Beltrametti-Cassinelli-Lahti model is modified so that it can describe a simplified ideal detector and corrected by adding a new rule, Rule 2 . It is based on empirical observations, in particular on the well-known fact that any individual registration yields a definite value (the objectification requirement). In the formulation of Rule 2, correlations play an important role. Appendix A describes the mathematical construction of $D$-local observables and Appendix B contains a proof that an entangled vector state of a composite system is completely determined by correlations between observables of a certain set.

The proposed Rule 2 is rather special and it is clear that a more general rule, or more rules, will be necessary to make quantum mechanics complete. This will require further work, both theoretical and experimental. This and other questions are discussed in the Conclusion.

\footnotetext{
${ }^{1}$ To prevent misunderstanding, let us mention that the term 'nonseparability' is sometimes used in a completely different sense (e.g., Ref. [11], p. 131) expressing the following valid property of quantum mechanics: a quantum state of a composite system contains more information than the sum of informations in the states of its constituents does.
} 


\section{Beltrametti-Cassinelli-Lahti Model of Quantum Measurement}

In this section, we are going to recapitulate the well-known ideas on measurement that will be needed later. A summary is [10], p. 25:

... the object system $\mathcal{S}$, prepared in a state $\mathrm{T}$ is brought into a suitable contacta measurement coupling-with another, independently prepared system, the measurement apparatus from which the result related to the measured observable $\mathrm{O}$ is determined by reading the value of the pointer observable.

In Ref. [10], these ideas are developed in detail with the help of models. One of them is as follows (p. 38). Let a discrete observable $\mathrm{O}$ of system $\mathcal{S}$ with Hilbert space $\mathcal{H}_{\mathcal{S}}$ be measured. Let $o_{k}$ be eigenvalues and $\left\{\phi_{k j}\right\}$ be the complete orthonormal set of eigenvectors,

$$
\mathrm{O} \phi_{k j}=o_{k} \phi_{k j}
$$

of $\mathrm{O}$. The projection $\mathrm{E}_{k}^{\mathrm{O}}$ on the eigenspace of $o_{k}$ is then $\mathrm{E}_{k}^{\mathrm{O}}=\sum_{j}\left|\phi_{k j}\right\rangle\left\langle\phi_{k j}\right|$. Let the registration apparatus ${ }^{2}$ be a quantum system $\mathcal{A}$ with Hilbert space $\mathcal{H}_{\mathcal{A}}$ and an observable $A$. Let $A$ be a non-degenerate, discrete observable with the same eigenvalues $o_{k}$ and with the complete orthonormal set of eigenvectors $\psi_{k}$,

$$
\mathrm{A} \psi_{k}=o_{k} \psi_{k}
$$

The projection on an eigenspace is $\mathrm{E}_{k}^{\mathrm{A}}=\left|\psi_{k}\right\rangle\left\langle\psi_{k}\right|$. A will be the pointer observable.

Let the measurement start with the preparation of $\mathcal{S}$ in state $\mathrm{T}$ and the independent preparation of $\mathcal{A}$ in state $\mathrm{T}_{\mathcal{A}}$. The initial state of the composed system $\mathcal{S}+\mathcal{A}$ is thus $\mathrm{T} \otimes \mathrm{T}_{\mathcal{A}}$.

Let $\mathcal{S}$ and $\mathcal{A}$ then interact for a finite time by the so-called measurement coupling and let the resulting state be given by $\mathrm{U}\left(\mathrm{T} \otimes \mathrm{T}_{\mathcal{A}}\right) \mathrm{U}^{\dagger}$, where $\mathrm{U}$ is a unitary transformation on $\mathcal{H}_{\mathcal{S}} \otimes \mathcal{H}_{\mathcal{A}}$.

The final state of the apparatus is $\operatorname{tr} \mathcal{S}\left[\mathrm{U}\left(\mathrm{T} \otimes \mathrm{T}_{\mathcal{A}}\right) \mathrm{U}^{\dagger}\right]$, where $t r_{\mathcal{S}}$ is the partial trace over states of $\mathcal{S}$. The first requirement on the model is that this state gives the same probability measure for the pointer observable as the initial state $T$ predicted for the observable O:

$$
\operatorname{tr}\left[\mathrm{TE}_{k}^{\mathrm{O}}\right]=\operatorname{tr}\left[\operatorname{tr} \mathcal{S}_{\mathcal{B}}\left[\mathrm{U}\left(\mathrm{T} \otimes \mathrm{T}_{\mathcal{A}}\right) \mathrm{U}^{\dagger}\right] \mathrm{E}_{k}^{\mathrm{A}}\right] .
$$

This is called probability reproducibility condition. Now, there is a theorem [14]:

Theorem 1 Let a measurement fulfil all assumptions and conditions listed above. Then, for any initial vector state $\psi$ of $\mathcal{A}$, there is a set $\left\{\varphi_{k l}\right\}$ of unit vectors in $\mathcal{H}_{\mathcal{S}}$ satisfying the orthogonality conditions

$$
\left\langle\varphi_{k l} \mid \varphi_{k j}\right\rangle=\delta_{l j}
$$

\footnotetext{
${ }^{2}$ In our language, a measurement consists of preparation and registration so that what Ref. [10] often calls 'measurement' is our 'registration'.
} 
such that $\mathrm{U}$ is a unitary extension of the map

$$
\phi_{k l} \otimes \psi \mapsto \varphi_{k l} \otimes \psi_{k}
$$

One assumes further that the eigenvalues of the pointer observable are uniquely associated with what will be read on the apparatus after the measurement. Then, the second requirement on the model is that it has to lead to a definite result. More precisely, the apparatus must be in one of the states $\left|\psi_{k}\right\rangle\left\langle\psi_{k}\right|$ after each individual registration. This is called objectification requirement. Reference [10] introduces a more general concept of measurement that leaves open whether the objectification requirement is satisfied or not. Such a procedure is called premeasurement. A measurement is then a premeasurement that satisfies objectification requirement.

Suppose that the initial state of $\mathcal{S}$ is an eigenstate, $\mathrm{T}=\left|\phi_{k l}\right\rangle\left\langle\phi_{k l}\right|$, with the eigenvalue $o_{k}$. Then, (1) implies that the final state of apparatus $\mathcal{A}$ is $\left|\psi_{k}\right\rangle\left\langle\psi_{k}\right|$, and the premeasurement does lead to a definite result. However, suppose next that the initial state is an arbitrary vector state, $\mathrm{T}=|\phi\rangle\langle\phi|$. Decomposing $\phi$ into the eigenstates,

$$
\phi=\sum_{k l} c_{k l} \phi_{k l}
$$

we obtain from (1)

$$
\mathrm{U}(\phi \otimes \psi)=\sum_{k} \sqrt{p_{\phi}^{\mathrm{O}}\left(o_{k}\right)} \Phi_{k} \otimes \psi_{k}
$$

where

$$
\Phi_{k}=\frac{\sum_{l} c_{k l} \varphi_{k l}}{\sqrt{\left\langle\sum_{l} c_{k l} \varphi_{k l} \mid \sum_{j} c_{k j} \varphi_{k j}\right\rangle}}
$$

and

$$
p_{\phi}^{\circ}\left(o_{k}\right)=\left\langle\sum_{l} c_{k l} \varphi_{k l} \mid \sum_{j} c_{k j} \varphi_{k j}\right\rangle
$$

is the probability that a registration of $\mathrm{O}$ performed on vector state $\phi$ gives the value $o_{k}$. The final state of apparatus $\mathcal{A}$ then is

$$
\operatorname{tr}_{\mathcal{S}}\left[\mathrm{U}\left(\mathrm{T} \otimes \mathrm{T}_{\mathcal{A}}\right) \mathrm{U}^{\dagger}\right]=\sum_{k l} \sqrt{p_{\phi}^{\mathrm{O}}\left(o_{k}\right)} \sqrt{p_{\phi}^{\mathrm{O}}\left(o_{l}\right)}\left\langle\Phi_{k} \mid \Phi_{l}\right\rangle\left|\psi_{k}\right\rangle\left\langle\psi_{l}\right|
$$

Because of the orthonormality of $\left|\psi_{k}\right\rangle$ 's, the probability that the apparatus shows the value $o_{k}$ if $\mathrm{A}$ is registered on it in this final state is $p_{\phi}^{\mathrm{O}}\left(o_{k}\right)$, which is what the probability reproducibility requires. However, if the objectification requirement is to be satisfied, two condition must be met:

(A) The final state of the apparatus must the convex combination of the form

$$
\operatorname{tr}_{\mathcal{S}}\left[\mathrm{U}\left(\mathrm{T} \otimes \mathrm{T}_{\mathcal{A}}\right) \mathrm{U}^{\dagger}\right]=\sum_{j} p_{\phi}^{\circ}\left(o_{j}\right)\left|\psi_{j}\right\rangle\left\langle\psi_{j}\right| .
$$


(B) The right-hand side of (5) must be the gemenge structure of the state.

The notion of gemenge will play an important role in the reasoning of the present paper. The term has been introduced in Ref. [10], some authors (e.g., Ref. [11]) use also the term 'proper mixture', Ref. [1,2] calls it 'direct mixture'. The crucial point is that the convex decomposition

$$
\mathrm{T}=\sum_{k=1}^{n} w_{k} \mathrm{~T}_{k}
$$

of any state $\mathbf{T}$ (state operator) can be a gemenge only if its preparation procedure $\mathbf{P}(\mathrm{T})$ is a random mixture with rates (frequencies) $w_{k}$ of preparations $\mathbf{P}\left(\mathrm{T}_{k}\right)$, where each $\mathbf{P}\left(\mathrm{T}_{k}\right)$ is some preparation procedure for $\mathrm{T}_{k}, k=1, \ldots, n$. The preparation mixture can be done by humans or result from some process in nature.

Thus, gemenge concerns a physical property of preparation rather than any mathematical one of the right-hand side of (6) (such as $\mathrm{T}_{k}$ being vector states or being mutually orthogonal, etc.). From the mathematical point of view, many different convex decompositions of a general state $T$ may exist. All possible components of such convex combinations form a so-called 'face' in the space of state operators (cf. [1], p. 75). A state is 'extremal' if it lies in a zero-dimensional face, that is, if it cannot be written as a non-trivial convex combination. Extremal states are described by projections onto one-dimensional subspaces of the Hilbert space. A preparation of T selects only one of the mathematically possible convex decompositions of $\mathrm{T}$.

A random mixture of preparations is not uniquely determined by the preparation process. It can be coarsened or refined, i.e., some of $\mathbf{P}\left(\mathrm{T}_{k}\right)$ can be combined into one preparation procedure or $\mathbf{P}\left(\mathrm{T}_{k}\right)$ for some $k$ can itself be a random mixture of other preparations.

Definition 1 The finest convex decomposition of state $\mathrm{T}$ defined by its preparation as gemenge is called gemenge structure of $\mathrm{T}$.

Thus, gemenge structure of $\mathrm{T}$ is uniquely determined by its preparation. For extremal states, there is always only one gemenge structure, the trivial one, independently of how it was prepared.

It may be advantageous to distinguish the mathematical convex combination of states from their gemenge structure by writing the sum in (6) as follows

$$
\mathrm{T}=\left(\sum_{k=1}^{n}\right)_{\mathrm{gs}} w_{k} \mathrm{~T}_{k}
$$

in the case that the right-hand side is a gemenge structure of $\mathrm{T}$.

The properties that follow directly from the definition of gemenge structure and that will be needed later are described by the following theorem. 


\section{Theorem 2}

1. Gemenge structure is preserved by unitary dynamics,

$$
\mathrm{U}\left(\sum_{k}\right)_{g s} w_{k} \mathrm{~T}_{k} \mathrm{U}^{\dagger}=\left(\sum_{k}\right)_{g s} w_{k} \mathrm{UT}_{k} \mathrm{U}^{\dagger}:
$$

if the sum on the left-hand side describes a gemenge structure of $\mathrm{T}$, then the gemenge structure of its evolution is described by the sum on the right-hand side.

2. In the following sense, gemenge structure is also preserved by composition of systems. Let $\mathrm{T}$ be a state of a composite system $\mathcal{S}+\mathcal{S}^{\prime}$. The necessary and sufficient condition for the partial trace over $\mathcal{S}^{\prime}$ to have the gemenge structure described by

$$
\operatorname{tr}_{\mathcal{S}^{\prime}}[\mathrm{T}]=\left(\sum_{k}\right)_{g_{s}} w_{k} \mathrm{~T}_{k}
$$

is that $\mathrm{T}$ itself has gemenge structure described by

$$
\mathrm{T}=\left(\sum_{k}\right)_{g s} w_{k} \mathrm{~T}_{k} \otimes \mathrm{T}_{k}^{\prime},
$$

where $\mathrm{T}_{k}^{\prime}$ are some states of $\mathcal{S}^{\prime}$.

All these ideas on gemenges seem to be well known. Now, an important new point will be added. In Ref. [12], we have accepted the non-objectivity of observables in its full extent, but we found a sufficient number of objective properties of quantum systems elsewhere. The summary of the ideas can be stated as follows

Objectivity Assumption A property is objective if its value is uniquely determined by a preparation according to the rules of standard quantum mechanics. The 'value' is the value of the mathematical expression that describes the property and it may be more general than just a real number. No registration is necessary to establish such a property but a correct registration cannot disprove its value; in many cases, registrations can confirm the value.

Objectivity Assumption led to a new realist interpretation of quantum mechanics, see the extended discussion in Ref. [12]. It leads also to a new meaning of gemenge structure: any individual system prepared in the state (7) is objectively in one of the states $\mathrm{T}_{k}$, because each of the systems has been prepared by one of the preparations $\mathbf{P}\left(\mathrm{T}_{k}\right)$, and the probability that $\mathbf{P}\left(\mathrm{T}_{k}\right)$ has been used is $w_{k}$.

Let us return to our point (B), which can now be written as

$$
\operatorname{tr}_{\mathcal{S}}\left[\mathrm{U}\left(\mathrm{T} \otimes \mathrm{T}_{\mathcal{A}}\right) \mathrm{U}^{\dagger}\right]=\left(\sum_{j}\right)_{\mathrm{gs}} p_{\phi}^{\mathrm{O}}\left(o_{j}\right)\left|\psi_{j}\right\rangle\left\langle\psi_{j}\right| .
$$

According to the meaning of the gemenge structure, this equation expresses the following property: after each registration, apparatus $\mathcal{A}$ is objectively in one of the states 
$\left|\psi_{j}\right\rangle\left\langle\psi_{j}\right|$ and it is in this state with probability $p_{\phi}^{\mathrm{O}}\left(o_{j}\right)$. This is exactly what objectification requirement is meant to be. Thus, the two points (A) and (B) can serve as an objectification criterion.

We can also understand why Beltrametti-Cassinelli-Lahti model of premeasurement does not satisfy the objectification criterion. Indeed, the end state $\mathrm{T} \otimes \mathrm{T}_{\mathcal{A}}$ of the system is $U(\phi \otimes \psi)(2)$, which is a vector state and can therefore have only a trivial gemenge structure. However, Point 2 of Theorem 2 implies that this is not compatible with state $\operatorname{tr}_{\mathcal{S}}\left[\mathrm{U}\left(\mathrm{T} \otimes \mathrm{T}_{\mathcal{A}}\right) \mathrm{U}^{\dagger}\right]$ being a non-trivial gemenge. Thus, we have shown a simple no-go theorem. An analogous difficulty holds for more general models of premeasurement described in Ref. [10] and the book contains more general no-go theorems. This is called problem of objectification. In fact, our theorem and main idea of proof are similar to those given in Ref. [10]. The rest of the paper will look for a reason why the vector state $\mathrm{U}(\phi \otimes \psi)$ must be replaced by a non-trivial gemenge so that the objectification criterion can be satisfied.

\subsection{Repeatable Premeasurement and Von-Neumann Model}

In order to define what a repeatable premeasurement is, we need the notion of state transformer. To this aim, let us first calculate the final state of system $\mathcal{S}$ after a Beltrametti-Cassinelli-Lahti premeasurement is finished:

$$
\operatorname{tr}_{\mathcal{A}}\left[\mathrm{U}(|\phi\rangle\langle\phi|\otimes| \psi\rangle\langle\psi|) \mathrm{U}^{\dagger}\right]=\sum_{k} p_{\phi}^{\mathrm{O}}\left(o_{k}\right)\left|\Phi_{k}\right\rangle\left\langle\Phi_{k}\right| .
$$

The part of the sum on the right-hand side corresponding to the result of premeasurement lying in the set $X$ is

$$
\mathcal{I}(X)(|\phi\rangle\langle\phi|)=\sum_{o_{k} \in X} p_{\phi}^{\mathrm{O}}\left(o_{k}\right)\left|\Phi_{k}\right\rangle\left\langle\Phi_{k}\right|
$$

The right-hand side is not a state, because it is not normalised. Its trace is the probability that the result lies in $X$,

$$
p_{\mathrm{\top}}^{\mathrm{O}}(X)=\operatorname{tr}[\mathcal{I}(X)(\mathrm{T})]
$$

if the initial state of $\mathcal{S}$ is $\mathrm{T}$. The quantity $\mathcal{I}(X)$ is an operation-valued measure and is called state transformer. For more details, see Ref. [10].

Definition 2 A premeasurement is called repeatable if its state transformer satisfies the equation

$$
\operatorname{tr}[\mathcal{I}(Y)(\mathcal{I}(X)(\mathrm{T}))]=\operatorname{tr}[\mathcal{I}(Y \cap X)(\mathrm{T})]
$$

for all subsets of possible values $X$ and $Y$ and all possible states $\mathrm{T}$ of $\mathcal{S}$.

That is, the repetition of the premeasurement on $\mathcal{S}$ does not lead to any new result from the probabilistic point of view. To see whether the state transformer (8) satisfies (9), let us rewrite it as follows: 


$$
\sum_{o_{k} \in X} p_{\phi}^{\mathrm{O}}\left(o_{k}\right)\left|\Phi_{k}\right\rangle\left\langle\Phi_{k}\left|=\sum_{o_{k} \in X} \mathrm{~K}_{k}\right| \phi\right\rangle\langle\phi| \mathrm{K}_{k}^{\dagger},
$$

where

$$
\mathrm{K}_{k}=\sum_{l}\left|\varphi_{k l}\right\rangle\left\langle\phi_{k l}\right|
$$

One can show that this relation is general,

$$
\mathcal{I}(X)(\mathrm{T})=\sum_{o_{k} \in X} \mathrm{~K}_{k} \mathrm{TK}_{k}^{\dagger},
$$

for proof, see Ref. [10]. We have then

$$
\mathcal{I}(Y)(\mathcal{I}(X)(\mathrm{T}))=\sum_{o_{l} \in X} \mathrm{~K}_{l}\left(\sum_{o_{k} \in X} \mathrm{~K}_{k} \mathrm{TK}_{k}^{\dagger}\right) \mathrm{K}_{l}^{\dagger}=\sum_{o_{l} \in X} \sum_{o_{k} \in X}\left(\mathrm{~K}_{l} \mathrm{~K}_{k}\right) \mathrm{T}\left(\mathrm{K}_{l} \mathrm{~K}_{k}\right)^{\dagger} .
$$

Equation (9) would be satisfied if

$$
\mathrm{K}_{l} \mathrm{~K}_{k}=\mathrm{K}_{k} \delta_{k l}
$$

which is in general not the case.

Let us therefore restrict ourselves to measurement couplings satisfying

$$
\phi_{k l}=\varphi_{k l} .
$$

This model is called von-Neumann premeasurement because it was first described in Ref. [16]. ${ }^{3}$

For von-Neumann premeasurement, the operator $\mathrm{K}_{k}$ is the projection $\mathrm{E}_{k}^{\mathrm{O}}$ on the eigenspace of $o_{k}$,

$$
\mathrm{K}_{k}=\sum_{l}\left|\phi_{k l}\right\rangle\left\langle\phi_{k l}\right|
$$

and (10) is satisfied. Thus, von-Neumann premeasurement is a special case of repeatable premeasurement.

The vector states $\Phi_{k}$ given by (3) are orthonormal for von-Neumann premeasurements. Thus, the final state of the apparatus given by (4) reduces to (5) and Point (A) of our objectification criterion is satisfied. As for Point (B), it is not satisfied even for the more general Beltrametti-Cassinelli-Lahti model of premeasurement. Hence, the objectification requirement does not hold for von-Neumann premeasurements, and it is therefore not a measurement.

Von Neumann himself postulated that measurements define another, non-unitary and indeterministic kind of evolution in which the state of $\mathcal{S}$ randomly jumps into one of the eigenstates of the measured observable (Ref. [16], pp. 217, 351). This was called collapse of the wave function by Bohm (Ref. [17], p. 120).

\footnotetext{
${ }^{3}$ In fact, von-Neumann premeasurement is slightly more general in the sense that it is a premeasurement of a function $f(\mathrm{O})$, where $f$ need not be bijective, cf. Ref. [10].
} 


\section{Comparison with Real Experiment. Importance of Detectors}

The theoretical models of the previous section ought to describe and explain at least some aspects of real experiments. This section will try to go into all experimental details that can be relevant to our theoretical understanding.

First, we briefly collect what we shall need about detectors. Microsystem $\mathcal{S}$ to be detected interacts with the sensitive matter of the detector so that some part of energy of $\mathcal{S}$ is transferred to the detector. Mostly, $\mathcal{S}$ interacts with many subsystems of the sensitive matter exciting each of them because the excitation energy is much smaller than the energy of $\mathcal{S}$. The resulting subsystem signals are collected, or amplified and collected so that they can be distinguished from noise. For example, in ionization detectors, many atoms or molecules of the sensitive matter are turned into electronion pairs. If the energy of $\mathcal{S}$ is much higher than the energy of one ionisation, e.g. about $10 \mathrm{eV}$, then many electron-ion pairs are produced and the positive as well as the negative total charge is collected at electrodes [18].

In the so-called cryogenic detectors [19], $\mathcal{S}$ interacts, e.g., with superheated superconducting granules by scattering off a nucleus and the phase transition from the superconducting into the normally conducting phase of only one granule leads to a perceptible electronic signal. A detector can contain very many granules (typically $10^{9}$ ) in order to enhance the probability of such scattering if the interaction between $\mathcal{S}$ and the nuclei is very weak (WIMP, neutrino). Modern detectors are constructed so that their signal is electronic. For example, to a scintillating film, a photomultiplier is attached, etc., see Ref. [18].

In any case, in order to make a detector respond $\mathcal{S}$ must loose some of its energy to the detector. The larger the loss, the better the signal. Thus, most detectors are built in such a way that $\mathcal{S}$ looses all its kinetic energy and is absorbed by the detector (in this way, also its total momentum can be measured). Let us call such detectors absorbing. If the bulk of the sensitive matter is not large enough, $\mathcal{S}$ can leave the detector after the interaction with it, in which case we call the detector non-absorbing. Observe that a detector is absorbing even if most copies of $\mathcal{S}$ leave the detector without causing a response but cannot leave if there is a response (e.g., neutrino detectors).

Suppose that $\mathcal{S}$ is prepared in such a way that it must cross a detector. Then, the probability of the detector response is generally $\eta<1$. We call a detector ideal, if $\eta=1$.

An important assumption, corroborated by all experiments, is that a real detector either gives a signal or remains silent in each individual registration. This corresponds here to the objectification requirement.

After these preparatory remarks, consider a typical repeatable premeasurement as described in textbooks (see, e.g., Ref. [15], p. 27, where it is called 'repeatable test'), for example a Stern-Gerlach-like measurement of spin. A coordinate system $\left\{x^{1}, x^{2}, x^{3}\right\}$ is chosen. Silver atoms evaporate in an oven $O$, form a beam $B_{0}$ along $x^{2}$-axis passing through a velocity selector $S$, and then through an inhomogeneous magnetic field produced by device $M_{1} . M_{1}$ splits $B_{0}$ into two beams, $B_{1+}$ and $B_{1-}$, of which $B_{1+}$ is associated with positive and $B_{1-}$ with negative spin $x^{1}$-component, the corresponding vector states being denoted by $|1+\rangle$ and $|1-\rangle$. Beam $B_{1-}$ is blocked off by a shield. This is the preparatory part of the experiment. 
Next, beam $B_{1+}$ runs through another magnetic device, $M_{3}^{(1)}$ with centre at $\vec{x}_{(1)}$ and finally strike an array of ideal detectors $\left\{D_{k}^{(1)}\right\}$ placed and oriented suitably with respect to $M_{3}^{(1)}$. Two detectors of array $\left\{D_{k}^{(1)}\right\}$ respond, let us denote them by $D_{+}$and $D_{-}$, revealing the split of $B_{1+}$ into two beams, $B_{3+}$ and $B_{3-}$, caused by $M_{3}^{(1)}$. Let the orientation of $M_{3}^{(1)}$ be such that $B_{3+}$ corresponds to positive and $B_{3}$ - to negative spin $x^{3}$-component, the states of silver atoms being $|3+\rangle$ or $|3-\rangle$. The beams $B_{3+}$ and $B_{3}$ - are spatially sufficiently separated so that their coordinates $\vec{x}_{3+}$ and $\vec{x}_{3-}$ at the point where they strike the detectors can be considered as classical values. In any case, they are measured by the detectors in a rather coarse-grained way. Let us call experiment I what is performed by $O, S, M_{1}, M_{3}^{(1)}$ and $\left\{D_{k}^{(1)}\right\}$.

Let us now remove $\left\{D_{k}^{(1)}\right\}$, place device $M_{3}^{(2)}$ of the same macroscopic structure and orientation as $M_{3}^{(1)}$ with centre position $\vec{x}_{(2)}$ in the way of $B_{3+}$ so that $B_{3}$ - passes by and arrange array $\left\{D_{k}^{(2)}\right\}$ so that it has the same relative position with respect to $M_{3}^{(2)}$ as $\left\{D_{k}^{(1)}\right\}$ had with respect to $M_{3}^{(1)}$. Now, only one detector will respond, namely that at the position $\vec{x}_{3+}-\vec{x}_{(1)}+\vec{x}_{(2)}$. Let us call experiment II what is performed by $O, S, M_{1}, M_{3}^{(1)}, M_{3}^{(2)}$ and $\left\{D_{k}^{(2)}\right\}$. The result of experiment II is described as 'two consecutive identical tests following each other with a negligible time interval between them ... yield identical outcomes' in Ref. [15].

Clearly, experiment II does not consist of two copies of experiment I performed after each other. The only repetition is that device $M_{3}^{(2)}$ is placed after $M_{3}^{(1)}$ and has the same structure and orientation with respect to its incoming beam $B_{3}$ as $M_{3}^{(1)}$ has with respect to $B_{1+}$. Device $M_{3}^{(1)}$ splits $B_{1+}$ into $B_{3+}$ and $B_{3-}$ but $M_{3}^{(2)}$ does not split $B_{3+}$. One may say that it leaves $B_{3+}$ unchanged. Let us define the action of device $M_{3}^{(k)}$ together with the choice of $( \pm)$-beam for each $k=1,2$ as a test (in the sense of Ref. [15]) or a premeasurements. Let the outcomes be the thought response of an imaginary detector placed in the way of the chosen beam. Then the (counterfactual) outcomes can be assumed to be identical indeed and we have an example of repeatable premeasurement that satisfies Definition 1.

The procedures defined in this way are premeasurements that can be described by von-Neumann model. The macroscopic positions $\vec{x}_{3+}$ or $\vec{x}_{3-}$ of the atom after it passes the magnet can be considered as the eigenvalues of the pointer observable associated with effects $|3 \pm\rangle\langle 3 \pm|$. However, the premeasurement cannot be considered as an instance of registration because it does not give us any information about the silver atoms. Try to suppose, e.g., that the arrangement measures effects $|3 \pm\rangle\langle 3 \pm|$ depending on which of the outgoing beams is chosen. Now, how can we recognise whether the outcome is 'yes' or 'not'? There is no change of a classical property of an apparatus due to its interaction with a microsystem that would indicate which of the values $\vec{x}_{3+}$ and $\vec{x}_{3}$ - results. But premeasurement is allowed not to give definite responses by each individual action. To obtain definite values, additional detectors are needed. Without the additional detector, however, this real premeasurement is not a measurement.

Suppose next that there are non-absorbing ideal detectors that do not disturb the spin state of the atom. This might work, at least approximately. Let experiment I' be the same as I with the only change that the array $\left\{D_{k}^{(1)}\right\}$ is replaced by $\left\{D_{k}^{p(1)}\right\}$ 
containing the non-absorbing detectors. Let experiment II' starts as I' and proceeds as II but with $\left\{D_{k}^{(2)}\right\}$ replaced by $\left\{D_{k}^{p(2)}\right\}$ made from the non-absorbing detectors. Clearly, the action of $\left(M_{3}^{(j)}+\left\{D_{k}^{p(j)}\right\}\right)$ for each $j=1,2$ is a repeatable premeasurement according to Definition 5 , and it is even a repeatable measurement because of the responses of the real detectors, but it definitely cannot be described by a vonNeumann theoretical model. For the detectors to response, some part of the energy of the atoms is needed, so that condition (11) is not satisfied.

An interesting difference emerges here between what we can say about the system (silver atom) on the one hand and about states on the other in their relation to the beams $B_{3+}$ and $B_{3-}$. Whereas $B_{3+}$ is associated with $|3+\rangle$ and $B_{3-}$ with $|3-\rangle$, each atom is in a linear superposition of the two states $|3+\rangle$ and $|3-\rangle$ that equals to the prepared state $|1+\rangle$. One can not even say that all atoms in beam $B_{3+}$ are in state $|3+\rangle$ because no atom is just in $B_{3+}$. Unlike the states, the atoms are not divided between the beams. Indeed, the two beams could be guided so that no detectors are in their two ways and that they meet each other again. Then, they would interfere and if the two ways are of equal length, so that no relative phase shift results, the original state $|1+\rangle$ would result. This would happen even if the beams are very thin, containing always at most one silver atom. Hence, each atom had to go both ways simultaneously.

Let us observe that each of the beams $B_{3+}$ and $B_{3-}$ by itself behave as if it were a prepared beam of silver atoms in a known state, which is $|3+\rangle$ and $|3-\rangle$, respectively. The voluntary element of beam choice in this experiment can be interpreted neither as a preparation, nor as a reselection of ensemble, nor as a collapse of the wave function. The fact that we place some arrangement $A$ of devices that do not contain any detector in the way of beam $B_{3}$ and leave $B_{3}$ - alone justifies our use of state in $|3+\rangle$ in all calculations of what will be the outcome after arrangement $A$ is passed. However, the whole outcome will be a linear superposition of states in each of the two beams at the time the upper beam passes $A$. Only if we put any detector after $A$ or, for that matter, a detector or just a shield into the way of $B_{3-}$, then something like a collapse of the wave function can happen. The arrangement with the shield in the way of $B_{3-}$ is a preparation of the vector state $|3+\rangle$.

The analysis of the present section motivates the following generalisation. First, an arrangement of devices that acts in agreement with von-Neumann model of premeasurement is neither a registration nor a preparation apparatus. Second:

Rule 1 Any registration apparatus for microsystems must contain at least one detector and every reading of an apparatus value is a signal from a detector.

If Rule 1 turns out not to be generally valid, then our theory of quantum measurement will work at least for those many cases in which it is.

\section{Cluster Separability}

Quantum systems of the same type are indistinguishable and this leads to entanglement. It seems then, that experiments with one particle might be disturbed by another 
particle of the same type, even if it were prepared independently, far away from the first. One can avoid similar problems by adding some assumption of locality to the axioms of quantum mechanics.

In the relativistic theory, one starts with the requirement that space-time symmetries of an isolated system (i.e., that is alone in space) be realised by unitary representations of Poincaré group on the Hilbert space of states, see Refs. [20] and [21]. Then, the cluster decomposition principle, a locality assumption, states that if multi-particle scattering experiments are studied in distant laboratories, then the $S$-matrix element for the overall process factorizes into those concerning only the experiments in the single laboratories. This ensures a factorisation of the corresponding transition probabilities, so that an experiment in one laboratory cannot influence the results obtained in another one. Cluster decomposition principle implies non-trivial local properties of the theory underlying the $S$-matrix, in particular it plays a crucial part in making local field theory inevitable (cf. Ref. [20], Chap. 4).

In the phenomenological theory of relativistic or non-relativistic many-body systems, Hilbert space of an isolated system must also carry a unitary representation of Poincaré or Galilei group. Then, the so-called cluster separability is a locality assumption, see, e.g., Refs. [22] or [23] and references therein. It is a condition on interaction terms in the generators of the space-time symmetry group saying: if the system is separated into disjoint subsystems (= clusters) by a sufficiently large spacelike separation, then each subsystem behaves as an isolated system with a suitable representation of space-time symmetries on its Hilbert space, see Ref. [22], Sect. 6.1. Let's call this principle cluster separability $I$.

Another special case of locality assumption has been described by Peres, Ref. [15], p. 128. Let us reformulate it as follows

Cluster Separability II No quantum experiment with a system in a local laboratory is affected by the mere presence of an identical system in remote parts of the universe.

It is well known (see, e.g., Ref. [15], p. 136) that this principle leads to restrictions on possible statistics (fermions, bosons). What is less well known is that it also motivates non-trivial locality conditions on states that can be prepared and on observables that can be registered.

The locality condition is formulated in Ref. [15], p. 128:

... a state $w$ is called remote if $\|A w\|$ is vanishingly small, for any operator A which corresponds to a quantum test in a nearby location. ... We can now show that the entanglement of a local quantum system with another system in a remote state (as defined above) has no observable effect.

This is a condition on $\mathrm{A}$ inasmuch as there has to be at least one remote state for $\mathrm{A}$.

However, Peres does not warn that the standard operators of quantum mechanics, which are in fact generators of space-time symmetries, do not satisfy his condition on A. Similarly, basic observables of relativistic-field or many-body theories are generators of Poincaré or Galilei groups and so they do not satisfy the locality condition, either. It follows that cluster separability II is logically independent from the cluster decomposition or of cluster separability I. Of course, this does not mean that the basic observables are to be rejected. They are very useful if the assumption of isolated sys- 
tem is a good approximation. However, it is definitely a bad one for quantum theory of measurement.

The present section expresses Peres' locality condition with the help of the socalled $D$-local observables. Based on this analysis, it then introduces the key notions of separation status and of its change. This is a modification of standard quantum mechanics that leads to a possibility of prescribing new rules for evolution of systems changing their separation status. Let us explain everything, working in $Q$ representation of the common Hilbert space $\mathbf{H}$ and of operators on it, which will be represented by their kernels. Then, one can also write tensor products as ordinary products and indicate the order of factors by indices at system coordinates.

Suppose that vector state $\psi\left(\vec{x}_{1}\right)$ of particle 1 is prepared in our laboratory as if no other particle of this type existed. Next, let vector state $\phi\left(\vec{x}_{2}\right)$ of particle 2 of the same type be prepared simultaneously in a remote laboratory. Then the state of the two particles must be

$$
\Psi\left(\vec{x}_{1}, \vec{x}_{2}\right)=\frac{1}{\sqrt{2}}\left(\psi\left(\vec{x}_{1}\right) \phi\left(\vec{x}_{2}\right) \pm \phi\left(\vec{x}_{1}\right) \psi\left(\vec{x}_{2}\right)\right)
$$

depending on the type statistics. If an observable with kernel $a\left(\vec{x}_{1} ; \vec{x}_{1}^{\prime}\right)$ is now measured in our laboratory, it is equally possible that the measurement is made on particle 1 or 2 and both can make a contribution to the outcome. Hence, the correct observable is described by two-particle kernel

$$
A\left(\vec{x}_{1}, \vec{x}_{2} ; \vec{x}_{1}^{\prime}, \vec{x}_{2}^{\prime}\right)=a\left(\vec{x}_{1} ; \vec{x}_{1}^{\prime}\right) \delta\left(\vec{x}_{2}-\vec{x}_{2}^{\prime}\right)+a\left(\vec{x}_{2} ; \vec{x}_{2}^{\prime}\right) \delta\left(\vec{x}_{1}-\vec{x}_{1}^{\prime}\right) .
$$

In our language, the composite system of the two particles is an object but each of the two particles is only a subobject. Thus, none of the particles possesses its standard set of states and standard set of effects. There is only a common one-particle Hilbert space, common standard set of one-particle states and common standard set of one-particle effects that the two particles share and that are formally equivalent to those of particle 1 if it were an object. These sets have only a formal, auxiliary significance. From the common Hilbert space, the physical Hilbert space of the composite system is formed by (anti)symmetrised tensor power containing states such as (12). From the formal point of view, $a\left(\vec{x}_{1} ; \vec{x}_{1}^{\prime}\right)$ (i.e., a $\left.\otimes 1\right)$ is not an operator on the (anti)symmetrised Hilbert space, but the operator (13) is. From the experimental point of view, the observable with kernel $a\left(\vec{x}_{1} ; \vec{x}_{1}^{\prime}\right)$ is not measurable but that with kernel (13) is.

There seems to be no control of states that are prepared anywhere in the world and the different possibilities have different measurable consequences. For example, the position of particle 1 as an object (i.e., without particle 2) has kernel $a\left(\vec{x}_{1} ; \vec{x}_{1}^{\prime}\right)=$ $\vec{x}_{1} \delta\left(\vec{x}_{1}-\vec{x}_{1}^{\prime}\right)$ and suppose that the position is measured. Then, the average is

$$
\int d^{3} x_{1} \vec{x}_{1} \psi^{*}\left(\vec{x}_{1}\right) \psi\left(\vec{x}_{1}\right) .
$$

On the other hand, the existence of particle 2 leads to the average

$$
\int d^{3} x_{1} d^{3} x_{2} d^{3} x_{1}^{\prime} d^{3} x_{2}^{\prime} \Psi^{*}\left(\vec{x}_{1}^{\prime}, \vec{x}_{2}^{\prime}\right) A\left(\vec{x}_{1}, \vec{x}_{2} ; \vec{x}_{1}^{\prime}, \vec{x}_{2}^{\prime}\right) \Psi\left(\vec{x}_{1}, \vec{x}_{2}\right)
$$




$$
=\int d^{3} x_{1} \vec{x}_{1} \psi^{*}\left(\vec{x}_{1}\right) \psi\left(\vec{x}_{1}\right)+\int d^{3} x_{1} \vec{x}_{1} \phi^{*}\left(\vec{x}_{1}\right) \phi\left(\vec{x}_{1}\right)
$$

The bigger the distance particle 2 has, the bigger the difference is.

Cluster separability II can now be stated as follows. The change of $\mathcal{S}_{1}$ state due to some actions in a remote laboratory would not be measurable if the wave-function support of the remote system did not intersects domain $D$ of the laboratory and if the observables that are measured were $D$-local in the following sense.

Definition 3 Let $a\left(\vec{x}_{1} ; \vec{x}_{1}^{\prime}\right)$ be an observable of $\mathcal{S}_{1}$, let $D$ be a domain of $\vec{x}_{1}$ and let

$$
\int d^{3} x_{1} a\left(\vec{x}_{1} ; \vec{x}_{1}^{\prime}\right) f\left(\vec{x}_{1}\right)=\int d^{3} x_{1}^{\prime} a\left(\vec{x}_{1} ; \vec{x}_{1}^{\prime}\right) f\left(\vec{x}_{1}^{\prime}\right)=0
$$

if ( $\operatorname{supp} f) \cap D=\emptyset$, where $f$ is a test function. Let us call such operators $D$-local.

Let us assume that $(\operatorname{supp} \psi) \subset D$ and $(\operatorname{supp} \phi) \cap D=\emptyset$. If $\mathcal{S}_{2}$ has been prepared and the $D$-local kernel $a_{D}\left(\vec{x}_{1}, \vec{x}_{1}^{\prime}\right)$ is used instead of $a\left(\vec{x}_{1} ; \vec{x}_{1}^{\prime}\right)$ in formula (13) defining operator $A_{D}$ instead of $A$ and we obtain

$$
\begin{gathered}
\int_{D} d^{3} x_{1} \int_{D} d^{3} x_{1}^{\prime} \int_{D} d^{3} x_{2} \int_{D} d^{3} x_{2}^{\prime} \Psi^{*}\left(\vec{x}_{1}, \vec{x}_{2}\right) A_{D}\left(\vec{x}_{1}, \vec{x}_{1}^{\prime} ; \vec{x}_{2}, \vec{x}_{2}^{\prime}\right) \Psi\left(\vec{x}_{1}^{\prime}, \vec{x}_{2}^{\prime}\right) \\
=\int_{-\infty}^{\infty} d^{3} x_{1} \int_{-\infty}^{\infty} d^{3} x_{1}^{\prime} \psi^{*}\left(\vec{x}_{1}\right) a\left(\vec{x}_{1} ; \vec{x}_{1}^{\prime}\right) \psi\left(\vec{x}_{1}^{\prime}\right)
\end{gathered}
$$

as if no $\mathcal{S}_{2}$ existed. It follows that in this case both rules for objects and rules for subobjects lead to the same results.

However, 'observables' that are usually associated with $\mathcal{S}_{1}$ are not $D$-local. For example, the position operator violates the condition by large margin, as seen above. In fact, the above analysis shows that such a 'position' is not measurable, be it represented by $\vec{x}_{1} \delta\left(\vec{x}_{1}-\vec{x}_{1}^{\prime}\right)$ or by $\vec{x}_{1} \delta\left(\vec{x}_{1}-\vec{x}_{1}^{\prime}\right)+\vec{x}_{2} \delta\left(\vec{x}_{2}-\vec{x}_{2}^{\prime}\right)$. Moreover, such an 'observable' controls position of the system in the whole infinite space. This is utterly different from observables that can be registered in a human laboratory. Nevertheless, one can modify any observable by a map called $\Lambda_{D}$ so that it becomes $D$-local and has the same averages in states with supports in $D$ as the original observable had, see Appendix A.

It seems, however, that a similar problem exists even if particle 2 is not remote: it can be prepared by a colleague on a neighbouring table in the same laboratory. Still, the experience shows that measurements done on particle 1 on the first table are not disturbed by the activity on the second table. Hence, the idea of cluster separability must work in the same way for a less remote case, too.

But now the extent of the whole problem comes to light. For simple microsystems, there are very many systems of the same type everywhere, at least according to our realist interpretation of quantum mechanics. Clearly, one could neglect the entanglement of a single microsystem $\mathcal{S}$ with all microsystems of the same type, if $\mathcal{S}$ had a non-trivial separation status in the following sense: 
Definition 4 Let $D$ be a domain and system $\mathcal{S}$ be prepared in a state with a $D$ local state operator T. Let the probability to register value of observable $\mathrm{E}(X)$ in set $X$ be $\operatorname{tr}[\operatorname{TE}(X)]$ for any $D$-local observable $\mathrm{E}(X)$ of $\mathcal{S}$. Then, domain $D$ is called separation status of $\mathcal{S}$.

Here, $\mathrm{T}$ is a $D$-local state operator and $\mathrm{E}(X)$ a $D$-local observable in the sense of Appendix A and the condition means that the registration of $\mathrm{E}(X)$ is not disturbed by any state different from $T$. We can then view such microsystems as physical objects.

For example, a microsystem that is alone in the Universe has separation status $D=\mathbb{R}^{3}$. This is a form of the assumption of isolated system. Measurable observables of such a system are the standard ones. The same microsystem in a domain $D$ but which is surrounded by matter containing a lot of microsystems of the same type such that supports of their states do not intersect $D$ has separation status $D$ and its measurable observables are the $D$-local ones. A trivial case of separation status for a microsystem is if the only available modus of description for it is that of a subobject. This has separation status $D=\emptyset$ and no observables of its own.

To formulate the idea of separation status mathematically, we allow an exception to the rule for composition of identical systems. Let system $\mathcal{S}$ be prepared in the separation status $D$ and let $\mathcal{S}^{\prime}$ be a family of $N$ systems of the same type as $\mathcal{S}$ in a domain $D^{\prime}, D \cap D^{\prime}=\emptyset$. Then the two systems $\mathcal{S}$ and $\mathcal{S}^{\prime}$ are to be composed according to the rule for composition of systems of different type. For example, let the wave function of $\mathcal{S}$ be $\psi(\vec{x})$ and that of $\mathcal{S}^{\prime}$ be $\Psi\left(\vec{x}_{1}, \ldots, \vec{x}_{N}\right)$ that is symmetric or anti-symmetric in its $N$ arguments according to the type. Then the wave function of composite system $\mathcal{S}+\mathcal{S}^{\prime}$ of $N+1$ subsystems of the same type must be written as

$$
\psi(\vec{x}) \Psi\left(\vec{x}_{1}, \ldots, \vec{x}_{N}\right) .
$$

Observe that wave function (15) is not (anti-)symmetric in all $N+1$ arguments! This is at variance with the formal prescription dealing with families of identical systems. According to this prescription, the wave function had to be

$$
\left[\psi(\vec{x}) \Psi\left(\vec{x}_{1}, \ldots, \vec{x}_{N}\right)\right]_{s, a},
$$

where the symbol $[\cdot]_{s, a}$ means symmetrisation or anti-symmetrisations over all wavefunction arguments contained inside. This modification of standard quantum mechanics is essential for our theory of measurement to work. Now, it also ought to be clear why we do not employ Fock-space method to deal with identical systems: it automatically (anti-)symmetrises over all systems of the same type.

The standard version of quantum mechanics as well as our interpretation [12, 13] of it can be understood as a theory of systems with a fixed status. Let us call these theories fixed status quantum mechanics (FSQM). They deal with individual microsystems according to one set of rules and with composite systems containing many particles of the same type according to another set of rules. It neglects the obvious relations between the two that make such an approach in principle inconsistent. However, the method seems to work and the justification why it approximately works is the cluster separability. It follows that FSQM has limits and that the limits have to do with the cases when separation status of system $\mathcal{S}$ changes. The main idea of the 
present paper is that there is certain freedom in choosing the state of $\mathcal{S}$ that results from a change of status (see Sect. 5).

The simplest example of separation status change is as follows. Suppose that wave function (15) is evolved further by the some first-quantised Hamiltonian according to prescriptions of standard quantum mechanics so that the support of wave function $\psi(\vec{x})$ changes from $D$ to $D^{\prime}$ (i.e., probability to find system $\mathcal{S}$ outside $D^{\prime}$ is then negligible) while $\Psi\left(\vec{x}_{1}, \ldots, \vec{x}_{N}\right)$ remains in $D^{\prime}$. Thus, the separation status of $\mathcal{S}$ becomes $\emptyset$ and $\mathcal{S}$ itself becomes a subobject. One possibility for the resulting state will now be described by (16), where the wave functions are replaced by their evolved versions. Observe that the change from state (15) to (16) is not unitary. This is in agreement with the fact that the set of observables measurable on $\mathcal{S}$ was radically reduced.

Let us close this section by a brief remark on macroscopic systems. In general, a macroscopic system $\mathcal{A}$ is a composite quantum system with very many different microsystem constituents. One can subdivide these microsystems into type classes. If we apply the basic rules of observable construction for systems of identical microsystems, then e.g. the position and momentum of any individual microsystem are not observables of $\mathcal{A}$. However, depending on how large the considered microsystem is and on the supports of all relevant states, some constituent microsystems can be considered as approximately separated. In general, to construct measurable observables for $\mathcal{A}$ is a non-trivial problem. For instance, eigenvalues of energy are not measurable (the spectrum of any macroscopic system is too dense for that). Instead, the average value of energy with some variance is measurable, etc., see Ref. [13]. Or, $X$-rays can be scattered by a crystal and so relative positions of its nuclei can be recognised. But rather than a position of an individual nucleus it is a space dependence of the average nuclear density due to all nuclei that is measured by the scattering.

\section{Gemenge Structure of Final Detector States}

Section 3 motivated the idea that the reading of a registration apparatus for microsystems is in fact an electronic signal from a detector. This gives us much clearer notion of registration apparatus. The main idea of Sect. 4 is that FSQM description of microsystems has its limits. This consequence of basic assumptions of standard quantum mechanics about indistinguishable microsystems leads to a significant modification of quantum theory of measurement. The necessary changes are:

1. Each preparation of microsystem $\mathcal{S}$ must separate the microsystem. Prepared state T must be $D$-local in a suitable domain $D$.

2. Microsystem $\mathcal{S}$ can then be manipulated and controlled by devices within $D$ such as electric and magnetic fields, matter shields, detectors, etc.

3. Let macrosystem $\mathcal{A}$ such as a blocking shield, a scattering target or a detector that contains microsystems indistinguishable from $\mathcal{S}$ lie in $D$. Corrections to FSQM description of the behaviour of the composed system $\mathcal{S}+\mathcal{A}$ due to a possible separation status change of $\mathcal{S}$ must be carefully chosen.

The usual method of FSQM is to specify initial states of both $\mathcal{S}$ and $\mathcal{A}$ before their interaction, choose some appropriate interaction Hamiltonian and calculate the corresponding unitary evolution of the composed system $\mathcal{S}+\mathcal{A}$ ignoring the problem with 
separation status change. As shown in Sect. 2, the results are wrong for registration apparatuses. We shall now try to choose some corrections.

Let $\mathcal{S}$ be the registered microsystem and $\mathcal{A}$ be an array of $N$ ideal monoatomic-gas ionisation detectors similar to that of Sect. 3. Let index $k$ enumerate the detectors and let each detector be treated as a system of identical atoms. Let each atom be modelled by a particle with mass $\mu$, spin zero and a further degree of freedom, ionisation, with two values, non-ionised and ionised. We simplify the model further by assuming that the ionisation and translation degrees of freedom can be separated from each other in such a way that they define two different formal subsystems, $\mathcal{A}_{\text {ion }}$ and $\mathcal{A}_{\text {tra }}$ of the whole real macroscopic system $\mathcal{A}$. Let $\chi_{k n}$ be the state describing $n$ ionised atoms in $k$ th detector. The states

$$
\prod_{k} \otimes \chi_{k n(k)}
$$

for all $n(k)$ 's form a basis of the Hilbert space of $\mathcal{A}_{\text {ion }}$, where $n(k)$ is a map of $\{1, \ldots, N\}$ into non-negative integers. Let us assume that the initial state of $\mathcal{A}_{\text {ion }}$ is

$$
\psi=\prod_{k} \otimes \chi_{k 0}
$$

the perfectly non-ionised state. We can further assume that the initial state $\mathrm{T}_{\text {tra }}$ of $\mathcal{A}_{\text {tra }}$ is close to maximum entropy one with sufficiently low temperature so that ionisations due to atomic collisions have a very low probability.

The measurement coupling is a coupling between $\mathcal{S}$ and the ionisation degree of freedom of each atom in the sensitive matter of the whole array. That is, $\mathcal{S}$ interacts directly only with $\mathcal{A}_{\text {ion }}$. In a single detector, after the ionisation of the first atom, all subsequent ionisations lie along a ray track inside the same detector. An explanation of the fact that e.g. a spherical wave can produce a straight track is given in Ref. [24], where it is shown that the position of the track head, the first ionisation of the track, determines the track. This can be considered as a necessary property of every measurement coupling that is possible in the case considered here. Let the measurement coupling be that of the Beltrametti-Cassinelli-Lahti model, satisfying (1), where

$$
\psi_{k}=\left(\prod_{j=1}^{k-1} \otimes \chi_{j 0}\right) \otimes\left(\sum_{n} a_{n} \chi_{k n}\right) \otimes\left(\prod_{j=k+1}^{N} \otimes \chi_{j 0}\right)
$$

and $a_{n}$ are coefficients independent of $k$ satisfying $\sum_{n}\left|a_{n}\right|^{2}=1$. This is again a simplifying assumption: each $\mathcal{S}$ creates always the same ionisation state in each detector.

In Sect. 2, states $\psi_{k}$ were called 'end states' of $\mathcal{A}$ and they were eigenstates of observable A called 'pointer observable'. Here, we prefer $\psi_{k}$ to be called trigger states because there is a further evolution of $\mathcal{A}$ independent of $\mathcal{S}$ that leads from $\psi_{k}$ to the concentration of charges at the electrodes, and an electronic signal, of $k$ th detector. This is due to a coupling between $A_{\text {ion }}$ and $A_{\text {tra }}$ mediated by the electrostatic field of the electrodes: ionised atoms move in a different way than the non-ionised ones. This motion leads to atom collisions and further ionisation in a complicated irreversible process. Only then, the true end states with true pointer values are achieved. There is 
no pointer observable, the pointer values being some averages with some variances, in agreement with the expectation of Refs. [12, 13]. However, what is important for us happens already at the trigger stage and we can ignore the evolution from a trigger state to a detector signal.

From the requirement that the measurement yields a definite result, an important statement follows (cf. Sect. 2):

Theorem 3 A measurement coupling of a true registration must be such that the end states $\varphi_{k l}$ of $\mathcal{S}$ are orthonormal,

$$
\left\langle\varphi_{k l} \mid \varphi_{m n}\right\rangle=\delta_{k m} \delta_{l n}
$$

The unitary evolution defined by the measurement coupling yields a trigger state of the whole system $\mathcal{S}+\mathcal{A}_{\text {ion }}$ given by (2). Then, the trigger state of $\mathcal{A}_{\text {ion }}$, obtained from (4) and (17), is given by (5).

According to Theorem 2, state (5) of $\mathcal{A}_{\text {ion }}$ has not the gemenge structure given by the right-hand side of (5) because of the entanglement with $\mathcal{S}$ due to state (2). The reason is that state (2) contains much more correlations between observables of $\mathcal{S}$ and $\mathcal{A}_{\text {ion }}$ than just correlations between the states $\Phi_{k}$ and $\psi_{k}$. To measure any of these correlations, we would always need some observables of $\mathcal{S}$ that do not commute with O (see Appendix B).

However, the assumption that the trigger state $\mathcal{A}_{\text {ion }}$ is (2) seems to be an illusion. Microsystem $\mathcal{S}$ is somewhere inside $\mathcal{A}$ at this stage and has become indistinguishable from other microsystems of the same type within $\mathcal{A}$. There is always a lot of them, either because they are present in the detectors before the registration started or because the detector becomes quickly polluted by them afterwards. Thus, the separation status of the system $\mathcal{S}$ has changed from an object to a subobject and with it also the separation status of the whole composite system $\mathcal{S}+\mathcal{A}$ has. The applications of FSQM to two systems of different separation status is different. In our case, system $\mathcal{S}+\mathcal{A}$ before the interaction is a composite one and each of the subsystems is an object having its states and observables. During and after the interaction, however, $\mathcal{S}$ ceases to be an object, becomes a part of $\mathcal{A}$ and looses all of its observables except of $O$. This is a deeper change than just a change of state. Hence, the existence of most correlations that are the content of state (2) is lost. The point is not that some observables are difficult to measure but rather that these observables do not exist at all. The only correlations that can remain are those between the trigger states $\psi_{k}$ of $\mathcal{A}_{\text {ion }}$ and $\Phi_{k}$ of the microsystem. They are the content of the state

$$
\sum_{k}\left|c_{k}\right|^{2}\left|\Phi_{k}\right\rangle\left\langle\Phi_{k}|\otimes| \psi_{k}\right\rangle\left\langle\psi_{k}\right|
$$

This motivates the following assumption:

Rule 2 Let a microsystem $\mathcal{S}$ be detected by a detector $\mathcal{A}$ and the measurement coupling satisfy (17) so that the corresponding unitary evolution leads to the state (2) 
with $\mathcal{S}$ inside $\mathcal{A}$. Then, instead of (2), the true state of $\mathcal{S}+\mathcal{A}_{\text {ion }}$ is

$$
\left(\sum_{k}\right)_{g s}\left|c_{k}\right|^{2}\left|\Phi_{k}\right\rangle\left\langle\Phi_{k}|\otimes| \psi_{k}\right\rangle\left\langle\psi_{k}\right| .
$$

It then follows from Theorem 2 that the trigger state of $\mathcal{A}_{\text {ion }}$ is

$$
\operatorname{tr}_{\mathcal{S}}\left[\mathrm{U}\left(\mathrm{T} \otimes \mathrm{T}_{\mathcal{A}}\right) \mathrm{U}^{\dagger}\right]=\left(\sum_{j}\right)_{\mathrm{gs}} p_{\phi}^{\mathrm{O}}\left(o_{j}\right)\left|\psi_{j}\right\rangle\left\langle\psi_{j}\right| .
$$

The content of Rule 2 is that only the correlations between the states $\psi_{k}$ of $\mathcal{A}_{\text {ion }}$ and $\Phi_{k}$ of the microsystem survive and all other correlations between $\mathcal{A}_{\text {ion }}$ and $\mathcal{S}$ are erased during the change of separation status of $\mathcal{S}+\mathcal{A}$. What survives and what is erased is uniquely determined by the Beltrametti-Cassinelli-Lahti model. In particular, the probability reproducibility condition determines states $\varphi_{k l}$ from the initial state $\psi$ of $\mathcal{A}_{\text {ion }}$ uniquely and the initial state $\phi$ of $\mathcal{S}$ determines states $\Phi_{k}$ uniquely. Thus, the additional evolution from state (2) to state (18) is non-unitary but still deterministic. Rule 2 is a new basic assumption which has to be added to quantum mechanics. To choose such an assumption, we have to look at observations and experiments. Rule 2 is in an agreement with what is observed.

A correct interpretation of Rule 2 distinguishes two cases. If the detectors are absorbing, then states $\Phi_{k}$ in (18) ought to be (anti-)symmetrised with states of other systems indistinguishable from $\mathcal{S}$ within the $k$-th detector as in (16). The expression $\left|\Phi_{k}\right\rangle\left\langle\Phi_{k}\right|$ in it just symbolises the fact that system $\mathcal{S}$ has been lost in the $k$-th detector. If they are non-absorbing, then state (18) contains states $\psi_{k}$ leading to detector signals on the one hand and describes the release of $\mathcal{S}$ in state $\Phi_{k}$ that is correlated with detector signals on the other. Each release is understood as an instance of preparation and the whole procedure is a random mixture of these single preparations. In both cases, the end state of $\mathcal{A}_{\text {ion }}$ is (19).

One can wonder whether a more detailed quantum mechanical model of what happens during a change of separation status can be constructed. The reason why this cannot be done within FSQM is that FSQM is not applicable to changes of separation status. Hence, a new law added to FSQM is needed.

As an example of a system of non-absorbing detectors, the MWPC telescope for particle tracking can be mentioned [18]. It is a stack of the so-called multiwire proportional chambers (MWPC), which is arranged so that a particle runs through exciting each of them. The resulting system of electronic signals contains the information about the particle track.

A registration by a non-absorbing detector is similar to a scattering of a microsystem by a macroscopic target. First, let us consider no-entanglement processes such as the scattering of electrons on a crystal of graphite with an interference pattern as a result [25] or the splitting of a laser beam by a down-conversion process in a crystal of $\mathrm{KNbO}_{3}$ (see, e.g., Ref. [26]). No-entanglement processes can be described by the following model. Let the initial state of the target $\mathcal{A}$ be $\mathrm{T}$ and that of the microsystem be $\phi$. We assume that the end state of the target is $T^{\prime}$ and the end-state of the microsystem is $\varphi$ and that we have a unitary evolution:

$$
|\phi\rangle\langle\phi|\otimes \mathrm{T} \mapsto| \varphi\rangle\langle\varphi| \otimes \mathrm{T}^{\prime} .
$$


There is no entanglement of the two systems due to the interaction and there is no necessity to divide the resulting correlations between $\mathcal{S}$ and $\mathcal{A}$ in what survives and what is erased. The end state is already of the form (18) and it has a trivial gemenge structure. In this way, our corrections of FSQM become trivial in this case.

A more complicated case is an entanglement scattering. Let microsystem $\mathcal{S}$ in initial state $\phi$ be scattered by a macrosystem $\mathcal{A}$ in initial state T and let this lead to excitation of different microscopic subsystems $\mathcal{S}_{k}^{\prime}$ of $\mathcal{A}$. Scattering of neutrons on spin waves in ferromagnets, transmutation of nuclei inside $\mathcal{A}$ or, for that matter, ionising an atom in a gas detector are examples. We have, therefore, a more general situation than that in which Rule 2 gives a unique result. It seems that the change of status must lead to some correlations between $\mathcal{S}$ and $\mathcal{A}$ surviving and some being erased. However, in this situation it must yet be investigated which is which. Clearly, the definitive general rule must depend on the two interacting systems and on the interaction Hamiltonian. More theoretical and experimental work is necessary to guess the general rule.

\section{Conclusion}

The present paper proposes some ideas based on cluster separability with the aim to solve the objectification problem of quantum measurement. Its main purpose is to show how the ideas work by studying well-understood, restricted class of physical conditions in which the following assumptions are a good approximation: (a) nonrelativistic quantum mechanics, (b) measurement performed directly on microsystems, (c) Beltrametti-Cassinelli-Lahti model of measurement and (d) pointer readings being signals from detectors.

Reference [10] defines and analyses the problem of objectification and shows its insolubility: no-go theorems such as Theorem 6.2.1, p. 76. One of the premises of all theorems of this kind is that standard quantum mechanics (without any further assumptions such as that of collapse of the wave function) is applicable to preparation and registration processes. The present paper gives a physical justification of why this premise is not valid: during preparation and registration, the system changes its separation status and standard quantum mechanics 1) is not applicable to, and 2) does not contain any rules for, such kind of evolution. Thus, new rules that govern changes of separation status can be added without any contradiction with standard quantum mechanics or proofs of no-go theorems. Rule 2 is an example of such a new rule. Thus, the no-go theorem of Sect. 2 is avoided.

An important result of the present paper together with Refs. [12,13] is a strongly improved understanding of preparation procedure. First, any preparation gives the prepared system its objective quantum properties such as states, gemenge structures, averages and variances of observables etc. so that it is justified to speak of a physical object. This is what we have called quantum object. Second, in certain sense, a preparation must separate a microsystem from the set of identical microsystems, at least approximately. Only then, it can be viewed as an individual system and the standard notion of observable becomes applicable to it. This is justified by the idea of cluster-separability. Third, a preparation must isolate the microsystem so that it can 
be individually manipulated by e.g. external fields or mater shields and registered by detectors.

One trend in the post-Everett theory of quantum measurement is to avoid the assumption of collapse of the wave function during registrations. In a sense, the present paper is heading in the opposite direction. We even replace the collapse by a more radical transformation, a change in microsystem description including state spaces and observable algebras. This change is, in plain words, a kind of loss of a registered object during its registration. However, our result for non-absorbing detectors and the old idea by von Neumann have some features in common.

After having shown that our ideas work under the simplified conditions listed above we can start thinking about extending the method to more general conditions. There is a lot of work to be done yet. First, we must turn to other models of measurement, for example to different (non-ideal) kinds of detectors or to the more realistic premeasurement models within the non-relativistic quantum mechanics. The main point is again that the state resulting from the evolution contains information about properties of the composite system $\mathcal{S}+\mathcal{A}$ that could be measured only if more observables than the registered one of $\mathcal{S}$ existed. Thus, a change of this illusory state analogous to that given by Rule 2 could be justified. In such a way, all no-go theorems could be defused. The exact division line between correlations that survive and those that are erased during the registrations and other processes might again be determined by a careful analysis of observational facts.

Next, relativistic corrections have been neglected so that all notions and rules of non-relativistic quantum mechanics could be used. An extension of the present results to relativistic fields seems to be a realistic project because cluster separability is valid in this field.

Acknowledgements The author is indebted to Štefan Jánoš for invaluable help with experimental physics and to Heinrich Leutwyler and Jiří Tolar for useful discussions. Thanks go to an anonymous reviewer for turning attention to the literature on many-body theory.

\section{Appendix A: Construction of $D$-local Observables}

For the construction, we need more mathematics. Let $\mathcal{L}_{r}(\mathcal{H})$ denote the set of all self-adjoint operators on the Hilbert space $\mathcal{H}$ that are bounded in the norm

$$
\|\mathrm{A}\|=\sup _{\|\psi\|=1}\|\mathrm{~A} \psi\|
$$

An operator $\mathrm{A} \in \mathcal{L}_{r}(\mathcal{H})$ is positive, $\mathrm{A} \geq 0$, where 0 is the null operator, if

$$
\langle\phi \mid A \phi\rangle \geq 0
$$

for all vectors $\phi \in \mathcal{H}$. The relation $\mathrm{A} \geq \mathrm{B}$ defined by

$$
A-B \geq 0
$$

is an ordering on this space. With this (partial) order relation, $\mathcal{L}_{r}(\mathcal{H})$ is an ordered Banach space. 
Definition 5 Let $\mathcal{F}$ be the Boolean lattice of all Borel subsets of $\mathbb{R}^{n}$. A positive operator valued (POV) measure

$$
\mathrm{E}: \mathcal{F} \mapsto \mathcal{L}_{r}(\mathcal{H})
$$

is defined by the properties

1. positivity: $\mathrm{E}(X) \geq 0$ for all $X \in \mathcal{F}$,

2. $\sigma$-additivity: if $\left\{X_{k}\right\}$ is a countable collection of disjoint sets in $\mathcal{F}$ then

$$
\mathrm{E}\left(\cup_{k} X_{k}\right)=\sum_{k} \mathrm{E}\left(X_{k}\right)
$$

where the series converges in weak operator topology, i.e., averages in any state converge to an average in the state.

3. normalisation:

$$
\mathrm{E}\left(\mathbb{R}^{n}\right)=1
$$

where 1 is the identity operator on $\mathcal{H}$.

The number $n$ is called dimension of $\mathrm{E}$. The operators $\mathrm{E}(X)$ for $X \in \mathcal{F}$ are called effects.

We denote by $\mathcal{L}_{r}(\mathcal{H})_{\leq 1}^{+}$the set of all effects.

Theorem $4 \mathcal{L}_{r}(\mathcal{H})_{\leq 1}^{+}$is the set of elements of $\mathcal{L}_{r}(\mathcal{H})$ satisfying the inequality

$$
0 \leq \mathrm{E}(X) \leq \mathrm{I}
$$

For the proof, see Ref. [1, 2].

A special case of POV measure is projection valued measure (PV measure). All effects of a PV measure are projections onto subspaces of $\mathcal{H}$. The spectral measure of a s.a. operator is a PV measure, hence POV measure is a generalisation of a s.a. operator. More about POV measures as well as the motivation for viewing them a quantum-mechanical observables, see Refs. [1, 2, 10, 15].

Let us denote by $\mathcal{H}_{D}$ the Hilbert space obtained by completion of $C^{\infty}$-functions with support in $D$ with respect to the scalar product of $\mathcal{H} . \mathcal{H}_{D}$ is a closed linear subspace of $\mathcal{H}$. Let $\mathrm{P}_{D}$ be the projection from $\mathcal{H}$ onto $\mathcal{H}_{D}$.

Definition 6 Let

$$
\Lambda_{D}: \mathcal{L}_{r}(\mathcal{H}) \mapsto \mathcal{L}_{r}(\mathcal{H})
$$

be defined by

$$
\Lambda_{D}(\mathrm{~A})=\mathrm{P}_{D} \mathrm{AP}_{D}
$$

Mapping $\Lambda_{D}$ is called $D$-localization. 
Clearly, $D$-localisation of any operator in $\mathcal{L}_{r}(\mathcal{H})$ is $D$-local. Everything that is measurable within $D$ can be described by $D$-local observables. Of course, the $D$ localisation is not a unitary map. For example, it does not preserve operator norm,

$$
\left\|\Lambda_{D}(\mathrm{~A})\right\| \leq\|\mathrm{A}\| \text {. }
$$

The operators and their $D$-localisations are considered as acting on $\mathcal{H}$. $D$-local operators leave $\mathcal{H}_{D}$ invariant and define, therefore, also operators on Hilbert space $\mathcal{H}_{D}$

We can use these facts in a construction of $D$-local POV measure on $\mathcal{H}_{D}$ from any observable $\mathrm{E}$ on $\mathcal{H}$ by $D$-localising the effects $\mathrm{E}(X)$. The normalisation condition becomes:

$$
\Lambda_{D}\left(\mathrm{E}\left(\mathbb{R}^{n}\right)\right)=\mathrm{P}_{D} 1 \mathrm{P}_{D}=1_{D},
$$

where $1_{D}$ is the identity operator on $\mathcal{H}_{D}$. Of course, $D$-localisation of a projection will not be a projection in general and so a $D$-localisation of a PV measure need not be a PV measure. Let us call this construction $D$-localisation of POV measures. All $D$-local POV measures commute with spectral projections of $\mathrm{PV}$ measure $\mathrm{E}^{\overrightarrow{\mathrm{Q}}}(X)$, if $X \cap D=\emptyset . \mathrm{E}^{\overrightarrow{\mathrm{Q}}}(X)$ is the spectral measure of the position operator $\overrightarrow{\mathrm{Q}}$. Thus, the restriction to $D$-local observables may be formally understood as superselection rules.

Everything can be easily extended from vector to general states; the state operators must just be $D$-local. If the map $\Lambda_{D}$ is involved in their construction it must be followed by a suitable normalisation.

\section{Appendix B: Complete Set of Correlations in a Vector State of a Composite System}

Consider a composite system with constituents $\mathcal{S}$ and $\mathcal{S}^{\prime}$ in vector state

$$
\Phi=\sum_{k} c_{k} \phi_{k} \otimes \phi_{k}^{\prime}
$$

$\left\{\phi_{k}\right\}$ being a basis of $\mathcal{H}_{\mathcal{S}},\left\{\phi_{k}^{\prime}\right\}$ that of $\mathcal{H}_{\mathcal{S}^{\prime}}$ and $c_{k}$ satisfying

$$
\sum_{k}\left|c_{k}\right|^{2}=1
$$

In fact, any vector state of $\mathcal{S}+\mathcal{S}^{\prime}$ can be written in the form (22), which is called Schmidt decomposition (see, e.g., [15], p. 123).

Let $\mathrm{O}$ be an observable of $\mathcal{S}$ and $\mathrm{O}^{\prime}$ of $\mathcal{S}^{\prime}$ and let us introduce the following abbreviations:

$$
\begin{aligned}
\langle\rangle\rangle_{\Phi} & =\langle\Phi|\mathrm{O} \otimes 1| \Phi\rangle, \\
\left\langle\mathrm{O}^{\prime}\right\rangle_{\Phi} & =\left\langle\Phi\left|1 \otimes \mathrm{O}^{\prime}\right| \Phi\right\rangle, \\
\left\langle\mathrm{OO}^{\prime}\right\rangle_{\Phi} & =\left\langle\Phi\left|\mathrm{O} \otimes \mathrm{O}^{\prime}\right| \Phi\right\rangle,
\end{aligned}
$$




$$
\begin{gathered}
\Delta_{\Phi} \mathrm{O}=\sqrt{\left\langle\mathrm{O}^{2}\right\rangle_{\Phi}-\langle\mathrm{O}\rangle_{\Phi}^{2}}, \\
\Delta_{\Phi} \mathrm{O}^{\prime}=\sqrt{\left\langle\mathrm{O}^{\prime 2}\right\rangle_{\Phi}-\left\langle\mathrm{O}^{\prime}\right\rangle_{\Phi}^{2}}
\end{gathered}
$$

The normalised correlation of $O$ and $O^{\prime}$ in $\Phi$ is defined by

$$
\rho\left(\mathrm{O}, \mathrm{O}^{\prime}, \Phi\right)=\frac{\left\langle\mathrm{OO}^{\prime}\right\rangle_{\Phi}-\langle\mathrm{O}\rangle_{\Phi}\left\langle\mathrm{O}^{\prime}\right\rangle_{\Phi}}{\Delta_{\Phi} \mathrm{O} \Delta_{\Phi} \mathrm{O}^{\prime}} .
$$

The normalised correlation always satisfies

$$
-1 \leqq \rho\left(\mathrm{O}, \mathrm{O}^{\prime}, \Phi\right) \leqq 1
$$

because of Schwarz' inequality. If $\rho\left(\mathrm{O}, \mathrm{O}^{\prime}, \Phi\right)=0$ observables $\mathrm{O}$ and $\mathrm{O}^{\prime}$ are uncorrelated, if $\rho\left(\mathrm{O}, \mathrm{O}^{\prime}, \Phi\right)= \pm 1$ they are strongly correlated/anti-correlated.

Let us first apply these formulae to projections,

$$
\mathrm{P}_{k}=\left|\phi_{k}\right\rangle\left\langle\phi_{k}\left|, \quad \mathrm{P}_{k}^{\prime}=\right| \phi_{k}^{\prime}\right\rangle\left\langle\phi_{k}^{\prime}\right| .
$$

Simple calculations yield

$$
\begin{gathered}
\left\langle\mathrm{P}_{k}\right\rangle_{\Phi}=\left\langle\mathrm{P}_{k}^{\prime}\right\rangle_{\Phi}=\left|c_{k}\right|^{2}, \\
\Delta_{\Phi} \mathrm{P}_{k}=\Delta_{\Phi} \mathrm{P}_{k}^{\prime}=\left|c_{k}\right| \sqrt{1-\left|c_{k}\right|^{2}}, \\
\left\langle\mathrm{P}_{k} \mathrm{P}_{l}^{\prime}\right\rangle_{\Phi}=\left|c_{k}\right|^{2} \delta_{k l} .
\end{gathered}
$$

Thus,

$$
\rho\left(\mathrm{P}_{k}, \mathrm{P}_{k}^{\prime}, \Phi\right)=1 .
$$

It follows that $\mathrm{P}_{k}$ and $\mathrm{P}_{k}^{\prime}$ are strongly correlated in $\Phi$.

Next, consider bounded, s.a. operators

$$
\begin{aligned}
& \mathrm{P}_{\alpha k l}=e^{i \alpha}\left|\phi_{k}\right\rangle\left\langle\phi_{l}\left|+e^{-i \alpha}\right| \phi_{l}\right\rangle\left\langle\phi_{k}\right|, \\
& \mathrm{P}_{\alpha k l}^{\prime}=e^{i \alpha}\left|\phi_{k}^{\prime}\right\rangle\left\langle\phi_{l}^{\prime}\left|+e^{-i \alpha}\right| \phi_{l}^{\prime}\right\rangle\left\langle\phi_{k}^{\prime}\right|
\end{aligned}
$$

for $k \neq l$. We calculate:

$$
\begin{gathered}
\left\langle\mathrm{P}_{\alpha k l}\right\rangle_{\Phi}=\left\langle\mathrm{P}_{\alpha k l}^{\prime}\right\rangle_{\Phi}=0, \\
\Delta_{\Phi} \mathrm{P}_{\alpha k l}=\Delta_{\Phi} \mathrm{P}_{\alpha k l}^{\prime}=\sqrt{\left|c_{k}\right|^{2}+\left|c_{l}\right|^{2}}, \\
\left\langle\mathrm{P}_{\alpha k l} \mathrm{P}_{\beta k l}^{\prime}\right\rangle_{\Phi}=e^{i(\alpha+\beta)} c_{k}^{*} c_{l}+e^{-i(\alpha+\beta)} c_{l}^{*} c_{k} .
\end{gathered}
$$

Thus,

$$
\rho\left(\mathrm{P}_{\alpha k l}, \mathrm{P}_{\beta k l}^{\prime}, \Phi\right)=\frac{e^{i(\alpha+\beta)} c_{k}^{*} c_{l}+e^{-i(\alpha+\beta)} c_{l}^{*} c_{k}}{\left|c_{k}\right|^{2}+\left|c_{l}\right|^{2}}
$$


It follows that correlations of the observables $\mathrm{P}_{\alpha k l}$ and $\mathrm{P}_{\beta k l}^{\prime}$ in state $\Phi$ contain complete information about all coefficient $c_{k}$ except for their common phase and so determine state $\Phi$. It is sufficient to use just two choices of $\alpha$ and $\beta$ :

1. $\alpha+\beta=0$,

2. $\alpha+\beta=\pi / 2$.

Next, consider state

$$
\mathrm{T}=\sum_{k}\left|c_{k}\right|^{2}\left(\left|\phi_{k}\right\rangle\left\langle\phi_{k}\right|\right) \otimes\left(\left|\phi_{k}^{\prime}\right\rangle\left\langle\phi_{k}^{\prime}\right|\right)
$$

For the projections $\mathrm{P}_{k}$ and $\mathrm{P}_{k}^{\prime}$, all averages in T equal to those in $\Phi$ and we have again

$$
\rho\left(\mathrm{P}_{k}, \mathrm{P}_{k}^{\prime}, \mathrm{T}\right)=1 \text {. }
$$

However, for the observables $\mathrm{P}_{\alpha k l}$ and $\mathrm{P}_{\alpha k l}^{\prime}$, we now obtain

$$
\begin{gathered}
\left\langle\mathrm{P}_{\alpha k l}\right\rangle_{\mathrm{T}}=\left\langle\mathrm{P}_{\alpha k l}^{\prime}\right\rangle_{\mathrm{T}}=0, \\
\Delta_{\mathrm{T}} \mathrm{P}_{\alpha k l}=\Delta_{\mathrm{T}} \mathrm{P}_{\alpha k l}^{\prime}=\sqrt{\left|c_{k}\right|^{2}+\left|c_{l}\right|^{2}}, \\
\left\langle\mathrm{P}_{\alpha k l} \mathrm{P}_{\beta k l}^{\prime}\right\rangle_{\mathrm{T}}=0 .
\end{gathered}
$$

Hence,

$$
\rho\left(\mathrm{P}_{\alpha k l}, \mathrm{P}_{\beta k l}^{\prime}, \mathrm{T}\right)=0 .
$$

Let us summarise: Correlations between $\mathrm{P}_{\alpha k l}$ and $\mathrm{P}_{\beta k l}^{\prime}$ determine state $\Phi$ uniquely. The change from $\Phi$ to T preserves the correlations between $\mathrm{P}_{k}$ and $\mathrm{P}_{k}^{\prime}$ but erases all correlations between $\mathrm{P}_{\alpha k l}$ and $\mathrm{P}_{\beta k l}^{\prime}$.

\section{References}

1. Ludwig, G.: Foundations of Quantum Mechanics I. Springer, New York (1983)

2. Ludwig, G.: Foundations of Quantum Mechanics II. Springer, New York (1985)

3. Ludwig, G.: An Axiomatic Basis for Quantum Mechanics 1. Springer, Berlin (1985)

4. Ludwig, G.: An Axiomatic Basis for Quantum Mechanics 2. Springer, Berlin (1987)

5. Giulini, D., Joos, E., Kiefer, C., Kupsch, J., Stamatescu, I.-O., Zeh, H.D.: Decoherence and the Appearance of Classical World in Quantum Theory. Springer, Berlin (1996)

6. Zurek, W.H.: Rev. Mod. Phys. 75, 715 (2003)

7. Hepp, K.: Helv. Phys. Acta 45, 237 (1972)

8. Primas, H.: Chemistry, Quantum Mechanics and Reductionism. Springer, Berlin (1983)

9. Bub, J.: Interpreting the Quantum World. Cambridge University Press, Cabridge (1999)

10. Bush, P., Lahti, P.J., Mittelstaed, P.: The Quantum Theory of Measurement. Springer, Heidelberg (1996)

11. d'Espagnat, B.: Veiled Reality. Addison-Wesley, Reading (1995)

12. Hájíček, P., Tolar, J.: Found. Phys. 39, 411 (2009)

13. Hájíček, P.: Found. Phys. 39, 1072 (2009)

14. Beltrametti, E.G., Cassinelli, G., Lahti, P.J.: J. Math. Phys. 31, 91 (1990)

15. Peres, A.: Quantum Theory: Concepts and Methods. Kluwer, Dordrecht (1995)

16. von Neumann, J.: Mathematical Foundation of Quantum Mechanics. Princeton University Press, Princeton (1983) 
17. Bohm, D.: Quantum Theory. Prentice-Hall, Englewood Cliffs (1951)

18. Leo, W.R.: Techniques for Nuclear and Particle Physics Experiments. Springer, Berlin (1987)

19. Twerenbold, D.: Rep. Progr. Phys. 59, 239 (1996)

20. Weinberg, S.: The Quantum Theory of Fields, vol. I, p. 177. Cambridge University Press, Cambridge (1995)

21. Haag, R.: Local Quantum Physics. Fields, Particles, Algebras. Springer, Berlin (1992)

22. Keiser, B.D., Polyzou, W.N.: In: Negele, J.W., Vogt, E. (eds.) Advances in Nuclear Physics, vol. 20. Plenum, New York (2002)

23. Coester, F.: Int. J. Mod. Phys. 17, 5328 (2003)

24. Mott, N.F.: Proc. R. Soc. Lond. A 126, 79 (1929)

25. Davisson, C., Germer, L.: Phys. Rev. 30 (1927)

26. Mandel, L., Wolf, E.: Optical Coherence and Quantum Optics. Cambridge University Press, Cambridge (1995) 